\title{
Zum Start der Zeitschrift: Was ist und welches Ziel verfolgt die Zeitschrift Coaching | Theorie und Praxis?
}

\author{
Eva-Maria Graf • Elke Berninger-Schäfer • Hansjörg Künzli
}

Online publiziert: 8. Juli 2015

(C) Die Autor(en) 2015. Dieser Artikel ist auf Springerlink.com mit Open Access verfügbar.

Nicht nur der Coaching-Markt, auch die wissenschaftliche Auseinandersetzung mit Coaching haben während der letzten Jahre eine exponentielle Entwicklung erlebt. Das verstärkte Interesse und Engagement an einer wissenschaftlichen Fundierung sind dabei eine vielfach gewünschte und wichtige Entwicklung, um die Professionalisierung und Qualitätssicherung von Coaching voranzubringen. So sind im Zeitraum von 2000-2004 im englischen Sprachraum ca. 12x mal mehr wissenschaftliche Artikel erschienen als im Zeitraum von 1995-1999. Für die Veröffentlichung und Diskussion ihrer Ergebnisse zum Thema Coaching stehen englischsprachigen Forschern und Forscherinnen dabei mehrere Journals wie das International Journal of Evidence Based Coaching and Mentoring oder Coaching: An International Journal of Theory, Research and Practice mit mehr oder weniger ähnlicher Ausrichtung zur Verfügung.

Der deutsche Sprachraum hat diese positive Entwicklung hin zu einer Professionalisierung von Coaching, wenn auch mit leichter Zeitverzögerung, ebenfalls vollzogen: Coaching ist mittlerweile auch in der deutschsprachigen Scientific Community angekommen und als relevantes Beratungsformat anerkannt; Coaching etabliert sich als eigenständiger Forschungsgegenstand in verschiedenen Disziplinen. Die Entwicklung von Praxis und Wissenschaft im Coaching im

E.-M. Graf $(\bowtie)$

Klagenfurt, Österreich

E-Mail: Eva-Maria.Graf@aau.at

E. Berninger-Schäfer

Karlsruhe, Deutschland

H. Künzli

Zürich, Schweiz

E-Mail: kasg@zhaw.ch deutschen Sprachraum ist dabei sehr dynamisch, innovativ und zunehmend unabhängig von anglo-amerikanischen Trends.

Was im deutschen Sprachraum allerdings bislang fehlte, war eine Zeitschrift, in der (primär) deutschsprachige wissenschaftliche Originalarbeiten mit einem ausschließlichen Fokus auf Coaching veröffentlicht werden konnten. Die Publikation eines Fachartikels in einem der englischsprachigen Journals ist zwar nach wie vor mit akademischen Meriten verbunden, erscheint aber oft als (unnötiger) Umweg zum Zielpublikum: Wissenschaftliche Artikel in englischer Sprache werden auch von wissenschaftlich interessierten Praktiker/innen kaum zur Kenntnis genommen, zumal - wie oben angedeutet - der Coaching-Markt, das Verständnis von Coaching etc. kulturelle Unterschiede aufweist und somit (theoretische) Erkenntnisse nicht einfach auf die deutschsprachige Coaching-Praxis übertragen werden können. Aber auch für die Wissenschaftler/innen selbst ist das Argumentieren im Hinblick auf Coaching als einem vom sozio-kulturellen Diskurs stark geprägten Phänomen innerhalb eben dieses Diskurses von großer Relevanz und zuweilen gewinnbringender als das notwendige Explizieren der jeweiligen kulturellen Ausprägung bzw. des kulturellen Verständnisses von Coaching im Zusammenhang mit einer Veröffentlichung in einem der englischsprachigen Journals.

Mit Coaching | Theorie \& Praxis gibt es nun ein Forum für diesen dynamischen und innovativen wissenschaftlichen Austausch auf dem deutschsprachigen Coaching(Forschungs-)Markt. Die Zeitschrift ist dabei zum einen primär in der Arbeits- und Organisationspsychologie beheimatet, zum anderen wird sie auf Grund des Forschungsgegenstandes auch interdisziplinäre Zugänge zum Thema fördern. So werden theoretische und empirische Original-Beiträge nicht nur aus der Psychologie, sondern auch der Soziologie, der Linguistik, der Beratungsforschung, 
der Betriebswirtschaftslehre sowie anderer Fachrichtungen, die sich wissenschaftlich mit dem Format Coaching befassen, berücksichtigt. Methodisch bietet die Zeitschrift sowohl quantitativen als auch qualitativen Ansätzen, aber auch Arbeiten basierend auf einem mixed method Ansatz eine Plattform, um die Bandbreite der wissenschaftlichen Zugänge zu Coaching aufzuzeigen und auch einen methodischen Dialog zu ermöglichen. Einen Dialog zwischen verschiedenen Perspektiven und Disziplinen im professionellen Coachingfeld schulenübergreifend $\mathrm{zu}$ ermöglichen, ist auch Zielsetzung des Deutschen Bundesverbandes Coaching (DBVC e. V.). Der DBVC enthält als einziger deutscher Coaching-Verband seit seiner Gründung 2004 die Säule Wissenschaft. Damit wurde frühzeitig signalisiert, dass die wissenschaftliche Fundierung der Coachingtätigkeit unerlässlich für die Professionsbildung und den Qualitätsdialog ist. Im Fachausschuss Forschung des DBVC ist die Idee für die Zeitschrift Coaching | Theorie \& Praxis entstanden.

\section{An wen richtet sich die Zeitschrift?}

Mit Coaching | Theorie \& Wissenschaft richten wir uns an Wissenschaftler/innen, die aus unterschiedlichen Disziplinen kommend zum Thema ,Coaching' forschen, um uns gegenseitig auf den neuesten Stand der Erkenntnisse zu bringen und auch den so wichtigen überdisziplinären Zugang zu Coaching zu befördern. Wir richten uns aber ebenso an wissenschaftlich interessierte Praktiker/innen aus den Bereichen Coaching, HR-Management, Personal- und Organisationsentwicklung sowie an Führungskräfte, die sich über aktuelle Forschungsansätze und -ergebnisse sowie die laufenden theoretischen Debatten informieren wollen. Das übergeordnete Ziel der Zeitschrift ist es, eine gemeinsame Wissensbasis zu schaffen, dieses Wissen laufend zu aktualisieren und in entsprechender Form zur Verfügung zu stellen, um so die Akademisierung, Professionalisierung und Qualitätssicherung im Coaching voranzubringen.

\section{Warum Theorie? Warum Praxis? Weil das Eine nicht ohne das Andere geht....}

Coaching kommt - im Unterschied zu anderen helfenden Interaktionen wie die Psychotherapie - aus der Praxis und ist fest in ihr verankert. Auch eine wissenschaftlich-theoretische Beschäftigung mit Coaching sollte daher diesen praktischen Wurzeln in ausreichendem Maße Rechnung tragen und praktische Überlegungen bzw. die praktische Relevanz der Fragestellungen mitberücksichtigen. Theoriebildung und Forschung (zu Coaching) soll und kann somit nicht im akademischen Elfenbeinturm stattfinden, sondern muss immer wieder den Austausch mit und die Anbindung an die Praxis suchen; umgekehrt muss sich die Praxis ihrerseits, will sie sich im Sinne einer reflexiven Praxis weiterentwickeln und ihre Qualität sicherstellen, kritisch mit ihrem eigenen Tun auseinandersetzen. Dazu aber bedarf es Modelle, Konzepte und einer Meta-Sprache, um eben diese Reflexion zu ermöglichen. Mit anderen Worten, es bedarf einer theoretisch-wissenschaftlichen Fundierung.

Eine theoretische und empirische Beschäftigung mit Coaching darf dabei aber nicht einer reinen Verifikationsfunktion dienen, die praktische Erkenntnisse primär bestätigt und ihnen dadurch eine (pseudo-)wissenschaftliche Legitimation verleiht. Theoriebildung und Forschung bedürfen immer (auch) der nötigen Distanz zum Forschungsgegenstand - in unserem Fall zu Coaching - um ihn auch kritisch hinterfragen zu können, in einen größeren sozio-kulturellen Zusammenhang zu stellen etc. und auf diese Weise neue, relevante - aber auch zu gegebener Zeit kritische - Erkenntnisse zu generieren.

\section{Warum Theorie?}

Theorien sind Bilder bzw. Modelle der Realität. Mittels theoretischer Aussagen können wir also die Realität - oder Ausschnitte daraus - beschreiben und/oder erklären und Prognosen über zukünftige Realitäten treffen. Auf Coaching übertragen: mit Hilfe adäquater Theorien können wir das, was im Coaching zwischen Coach und Klient oder Klientin passiert, beschreiben, erklären und (bis zu einem gewissen Grad) vorhersagen. Theorien können Aufschluss darüber geben, warum bestimmte Interventionen wirken, unter welchen Bedingungen sich Menschen verändern (können), welchen impliziten „Regeln“ Kommunikation folgt usw. Theorien bilden sich dabei jeweils um bzw. zu einem Gegenstand als einem Ausschnitt der Realität. Die Theoriebildung und Forschung zum Gegenstand ,Coaching ' wird dabei im deutschsprachigen Raum disziplinär weitergefasst und eher als ein Teil einer sich formierenden Beratungswissenschaft verstanden. Im Unterschied dazu wird im englischsprachigen Raum Coaching-Forschung oftmals mit Coaching-Psychologie gleichgesetzt.

Im Zusammenhang mit Coaching muss die Theoriebildung und Forschung als ersten wesentlichen Schritt ihren Gegenstand selbst definieren und konkretisieren. Dies ist umso wichtiger, da Coaching oftmals zu Recht als unspezifischer Container-Begriff kritisiert wurde, wobei die Verwendung des Begriffs, Coaching teilweise wahllos für alle möglichen Arten von Beratung geschieht. An dieser Stelle soll daher die unserer Zeitschrift sowie im Großen und Ganzen auch den Beiträgen zugrundeliegende, mittlerweile etablierte, Definition von Coaching formuliert werden: 
Unter Coaching verstehen wir ein Beratungsformat, das primär prozessorientiert ausgerichtet ist und sich an Personen im Arbeitskontext richtet, die ihre Handlungsfähigkeit wiederherstellen oder erweitern wollen und professionelle Begleitung durch einen externen oder auch internen Coach bei der (eigenen) Erarbeitung von Lösungen und Zielen suchen. Das übliche Setting dafür ist das Einzelcoaching, Gruppen- und Teamsettings werden aber hier ebenfalls mitberücksichtigt. Während sich Coaching dabei ursprünglich auf die Begleitung von Führungskräften, vornehmlich im Einzelsetting bezog, gibt es inzwischen eine Vielfalt von unterschiedlichen Coachingformaten und Zielgruppen. Diese Ausdifferenzierung von CoachingFormaten hängt mit der zunehmenden Bedeutung und Bekanntheit von Coaching einerseits und mit Veränderungen in der Arbeitswelt andererseits zusammen.

\section{Warum Praxis?}

Wie bereits gesagt, war und ist Coaching ein Kind der Praxis. Einblicke in die Coaching-Praxis aus Sicht praktizierender Coachs finden sich in den äußerst zahlreichen Coaching-Büchern auf dem Markt. Im Zusammenhang mit Coaching geht es als nicht darum, Konzepte und Modelle für einen entstehenden Gegenstand zu generieren, sondern eher darum, für eine existierende und etablierte Praxis adäquate Modelle und Konzepte zu ihrer Beschreibung $\mathrm{zu}$ entwickeln, d. h. also eine theoretische Fundierung und Modellbildung sowie eine empirische Auseinandersetzung mit diesen Theorien ex post zu begründen. Benötigt werden somit mehr theoretische Erkenntnisse und empirische Ergebnisse, um das ,know that' der Praktiker/innen durch ein ,know how' zu ergänzen, aber eben auch kritisch zu hinterfragen, weiterzuentwickeln, zu evaluieren etc. Um die Praxisrelevanz zu gewährleisten, die nicht zu verwechseln ist mit Affirmationsdiensten bzw. Auftragsforschung, bedarf es einer engen Zusammenarbeit und eines kontinuierlichen Austausches zwischen Forschung und Praxis. Ideal sind dabei interprofessionelle Kompetenzen der Beteiligten, die es erlauben Objekte in Subjekte zu übertragen: aus einem Objekt der Forschung, wie z. B. dem/der Coach bzw. seinem/ihrem professionellen Handeln, wird ein Subjekt der Forschung, indem der/die Coach am Forschungsaufbau oder -prozess etc. aktiv beteiligt ist.

Die Realität der Zusammenarbeit von Coaching-Forschung und Coaching-Praxis stellt sich allerdings komplexer und schwieriger dar und die vorliegende Zeitschrift sieht ihre Aufgabe daher auch darin, als vermittelnde Instanz eine Brücke zu schlagen. Dabei gilt es u.a. sich den folgenden Herausforderungen bezüglich einer fruchtbringenden Zusammenarbeit von Theorie und Praxis zu stellen:

- Zunächst gilt es innerhalb der Coaching-Forschung, die ja in verschiedenen Fachrichtungen angesiedelt und somit stark inter- bzw. eigentlich transdisziplinär geprägt ist, sich der mannigfachen und oftmals stark unterschiedlichen Annahmen, methodischen Herangehensweisen, Forschungstraditionen etc. bewusst zu werden und den Austausch und die Reflexion darüber zu fördern.

- Es gilt desweitern eine gemeinsame Sprache der Verständigung und des Austausches zwischen Coaching-Forschung und Coaching-Praxis im Sinne einer Meta-Sprache zu entwickeln. Wichtig ist dabei auch, sich möglicher unterschiedlicher Lesarten bzw. zugrunde liegender Konzepte bei vermeintlich gleichen Begrifflichkeiten bewusst zu werden um Missverständnisse zu vermeiden.

- Es gilt die unterschiedlichen Anliegen und Aufträge von Wissenschaft und Praxis zu berücksichtigen: Während die Wissenschaft zum Zwecke des Erkenntnisgewinns zunächst kompliziert und detailliert, gibt es in der Praxis das starke Bedürfnis nach einfachen, praktisch umsetzbaren Modellen.

- Es gilt auch die unterschiedlichen Perspektiven von praktizierenden und forschenden Coachs, von internen und externen Coachingpool-Anbietern und CoachingWeiterbildungsanbietern (akademische und nicht akademische) zu berücksichtigen. D. h. die Coaching-Praxis ist in ihrer jeweiligen Unterschiedlichkeit und Komplexität wahrzunehmen. Aber auch die Seite der Wissenschaft bzw. der Coaching-Forschung ist neben der bereits erwähnten Inter- bzw. Transdisziplinarität sowohl im Sinne einer Grundlagenforschung als auch im Sinne einer angewandten oder anwendungsorientierten Forschung mit ihren jeweils unterschiedlichen Aufträgen und Herangehensweisen zu differenzieren.

Das gemeinsame Interesse aller unterschiedlichen Perspektiven sollte in der Professionalisierung und der damit zusammenhängenden Qualitätssicherung von Coaching liegen. Hierfür ist der Brückenschlag zwischen Theorie und Praxis, zwischen Wissenschaft und Anwendung unerlässlich, damit Coaching angesichts gesellschaftlicher, politischer und wirtschaftlicher Veränderungen zukunftsfähig bleibt: So wie sich das Führungsverständnis durch sich verändernde Werte im Arbeitsleben und einer bestimmten Erwartungshaltung der jüngeren Generation entsprechend verändern muss, um zukunftsfähig zu sein, stellt sich im Besonderen auch die Frage nach der Zukunftsfähigkeit von Coaching. Dies ist eine außerordentlich bedeutsame Fragestellung sowohl für die Coaching-Forschung als auch insbesondere für die Coaching-Praxis. 
Das Team der Herausgeber/innen spiegelt unseren Anspruch der Zeitschrift und unsere Grundidee wider, Coaching Disziplinen- und Methoden-übergreifend zu erforschen und stets den Bezug zur Coaching-Praxis im Auge zu haben:

Dr. Elke Berninger-Schäfer ist promovierte Psychologin, Senior Coach des DBVC, Senior Coach und Lehrcoach der Führungsakademie Baden-Württemberg und Inhaberin des Karlsruher Instituts für Coaching, Personal- und Organisationsentwicklung. Mit verschiedenen Kooperationspartnern aus Wirtschaft, Wissenschaft und Verwaltung bildet sie Coachs in Kontakt- und Masterstudiengängen aus. Sie leitet den Fachausschuss Forschung des DBVC.

Prof. Dr. Eva-Maria Graf ist Assoziierte Professorin in der Angewandten Sprachwissenschaft an der Alpen-Adria Universität in Klagenfurt und habilitierte sich zum Diskurs im Coaching aus linguistischer Sicht. Sie ist Mitbegründerin der interdisziplinären Forschungsgruppe LOCCS (The Linguistics of Coaching, Consulting and Supervision), Mitglied im Fachausschuss Forschung des DBVC, tätig im Coaching-Programm der Universität St. Gallen und arbeitet als Coach und Lehrcoach.

Professor Hansjörg Künzli ist Professor für angewandte Psychologie an der Züricher Hochschule für Angewandte Wissenschaften. Er leitet dort die Forschungsschwerpunkt Beratung, Training und Führung, lehrt zu Forschungsmethoden und Motivation und forscht in den Bereichen Coaching, Berufslaufbahnberatung, Kommunikationsberatung und Training.

Auch durch den Beirat unserer Zeitschrift (siehe www. springer.com/40896) wird unser Anspruch an eine breite wissenschaftliche und methodische Basis sowie einen aktuellen Bezug zur Coaching-Praxis verdeutlicht.

\section{Aufbau und Dissemination der Zeitschrift}

Die Zeitschrift Coaching | Theorie \& Praxis veröffentlicht (primär) deutschsprachige Originalartikel aus allen Disziplinen, die sich wissenschaftlich mit dem Forschungsgegenstand Coaching befassen. Da es sich um ein transdisziplinäres Projekt handelt, wird den unterschiedlichen Wissenschaftstraditionen insofern Rechnung getragen, als die Artikel nur im Sinne von theoretisch oder empirisch und bezüglich einer quantitativen, qualitativen oder mixed method Methode unterschieden werden, ansonsten wird keine Kategorisierung vorgenommen. Alle Beiträge durchlaufen ein double blind Review Verfahren, d. h. weder weiß der/die Reviewer, wessen Artikel er/sie begutachtet, noch weiß der/die Autor/in des Beitrags von wem sein/ihr Beitrag begutachtet wird. Um eine kritische Auseinandersetzung mit dem Beitrag zu gewährleisten, wird auf disziplinäre Nähe im Begutachtungsprozess geachtet.

Coaching | Theorie \& Praxis wird als sog. Fully Funded Open Access Journal erscheinen und ist als solches die erste ihrer Art auf dem deutschsprachigen Markt. CTP wird vollständig von ihren Sponsoren, dem DBVC, der ZHAW und der Führungsakademie Baden Württemberg finanziert und kann kostenfrei über das Internet abgerufen werden. Sie steht somit allen Interessierten frei zur Verfügung. Wir betrachten diese moderne Form der Publikation als wesentlichen Vorteil:Es handelt sich um eine zeitgemäße Verbreitung von Wissen und um eine wichtige Demokratisierung des Wissens; gerade Praktiker/innen verfügen nicht über einen Zugang zu einer Hochschulbibliothek und der Preis von wissenschaftlichen Zeitschriften ist aus Sicht der Praktiker/innen prohibitiv hoch.

Als Fully Funded Open Access Journal erscheint Coaching | Theorie \& Praxis nicht in Form einzelner bzw. ganzer Ausgaben. Stattdessen werden die Beiträge laufend online unter der Marke Coaching | Theorie \& Praxis veröffentlicht, was den Leser/innen ein Höchstmaß an Aktualität der Erkenntnisse garantiert und den Beitragenden den unmittelbaren Austausch mit der Scientific Community erlaubt.

Danksagung Wir wollen wir uns beim Springer Verlag und unserem Verleger Herrn Dr. Klockenbusch für die hilfreiche und effiziente Unterstützung bei der Realisierung unseres Projekts und den anfallenden Arbeiten für diese erste Ausgabe bedanken!

Ebenfalls ganz herzlich wollen wir uns bei unseren Mitarbeiterinnen Sandra Schwyter und Anne Lohmann von der Züricher Hochschule für Angewandte Wissenschaften für die tatkräftige und kompetente Unterstützung bedanken!

Open Access Dieser Artikel wird unter der Creative Commons Namensnennung 4.0 International Lizenz (http://creativecommons.org/licenses/ by/4.0/deed.de) veröffentlicht, welche die uneingeschränkte Nutzung, Verbreitung und Wiedergabe für beliebige Zwecke erlaubt, sofern Sie den/die ursprünglichen Autor(en) und die Quelle ordnungsgemäß nennen, einen Link zur Creative Commons Lizenz beifügen und angeben, ob Änderungen vorgenommen wurden. 